\title{
A Novel MADM Approach Based on Fuzzy Cross Entropy with Interval-Valued Intuitionistic Fuzzy Sets
}

\author{
Xin Tong and Liying Yu \\ School of Management, Shanghai University, Shanghai 200444, China \\ Correspondence should be addressed to Liying Yu; yuliying@shu.edu.cn
}

Received 16 October 2014; Revised 24 December 2014; Accepted 25 December 2014

Academic Editor: Guangming Xie

Copyright ( 2015 X. Tong and L. Yu. This is an open access article distributed under the Creative Commons Attribution License, which permits unrestricted use, distribution, and reproduction in any medium, provided the original work is properly cited.

\begin{abstract}
The paper presents a novel multiple attribute decision-making (MADM) approach for the problem with completely unknown attribute weights in the framework of interval-valued intuitionistic fuzzy sets (IVIFS). First, the fuzzy cross entropy and discrimination degree of IVIFS are defied. Subsequently, based on the discrimination degree of IVIFS, a nonlinear programming model to minimize the total deviation of discrimination degrees between alternatives and the positive ideal solution PIS as well as the negative ideal solution (NIS) is constructed to obtain the attribute weights and, then, the weighted discrimination degree. Finally, all the alternatives are ranked according to the relative closeness coefficients using the extended TOPSIS method, and the most desirable alternative is chosen. The proposed approach extends the research method of MADM based on the IVIF cross entropy. Finally, we illustrate the feasibility and validity of the proposed method by two examples.
\end{abstract}

\section{Introduction}

The multiple attribute decision-making (MADM) is a common approach to modeling in the social science, economics, management, decision making, and some other multidiscipline fields. The MADM is based on a set of theories, methods, and procedures, which are used to solve the problem of determining a reasonable or optimal alternative from a set of alternatives faced by the decision maker.

Since the introduction of the fuzzy sets (FS) theory by Zadeh [1], it has been successfully applied to various fields. However, the traditional FSs cannot fully describe information in some research problems, such as determining to which set the membership degree of an element belongs. Another example of the limitations of the traditional FS theory is that it cannot work with membership degrees containing certain hesitation or uncertainty. As a result of these limitations, Atanassov [2] developed the concept of intuitionistic fuzzy sets (IFS) in 1986, which generalized the FS theory. An IFS can be described by a membership degree and nonmembership degree, which is more flexible and practical than the standard FS concept. Today the IFS theory is widely used in problems dealing with vagueness and uncertainty.
In the MADM, due to the usual complexity and uncertainty of the objective world, as well as the limitations of the knowledge level, and asymmetry and fuzziness of the information obtained from different decision makers (DM), sometimes it is hard to use an accurate real value to describe the membership and nonmembership degrees of IFS. As a result, the membership and nonmembership degrees often take the form of interval numbers. Atanassov and Gargov [3] introduced the concept of interval-valued intuitionistic fuzzy sets (IVIFS) based on the extended IFS theory, which uses interval numbers instead of real numbers to denote the membership and nonmembership degrees. IVIFS theory improves the ability of expressing the uncertainty of information.

In recent years, with the rapid development of the social economy and increased volume of information, the application of the MADM to real world problems has become more complex, obscure, and uncertain. Many theories and methods of IFS and IVIFS have been developed and extensively applied to solve the fuzzy MADM problems, such as group evaluation [4], logic programming [5], medical diagnosis $[6,7]$, and pattern recognition $[6,8]$.

In the fuzzy set theory, the entropy as a measure of fuzziness was first used by Zadeh [9], which has become a 
very important notion of measuring the degree of fuzziness or uncertainty of information [10]. The starting point of the cross-entropy approach is the information theory developed by Shannon [11]. Kullback and Leibler [12] proposed a measure of the "cross-entropy distance" between two probability distributions. Shang and Jiang [13] developed the fuzzy cross entropy, which was used to denote the symmetric discrimination information measure between two FSs. Vlachos and Sergiadis [14] introduced the concepts of the intuitionistic fuzzy cross entropy and discrimination information for IFS. Then Ye [15] developed a MADM method based on the intuitionistic fuzzy cross entropy (IF cross entropy). Moreover, Ye [16] introduced the concept of the intervalvalued intuitionistic fuzzy cross entropy (IVIF cross entropy), analogous to the IF cross entropy.

Although the entropy has drawn the attention of many researchers, not much effort has been paid to investigate the application of the IVIF cross entropy, or to avoid information loss in the process of information aggregation. In particular, the research related to the direct usage of the IVIF cross entropy to determine attribute weights and rank alternatives is at a relatively early stage of development. The goal of the paper is to propose a novel approach to the MADM problem based on the IVIF cross entropy used to rank the alternatives and choose the best alternative, where the information about attribute weights for alternatives is completely unknown and the decision matrix is defined in terms of IVIFSs.

The rest of the paper is organized as follows. In Section 2, a brief introduction to the IFS and IVFS theory and the fuzzy cross entropy is given. In Section 3, a novel approach to the MADM problem based on the IVIF cross entropy is proposed, which allows determination of the completely unknown attribute weights using both the positive ideal solution (PIS) and negative ideal solution (NIS) as the object of reference, to apply the extended TOPSIS to rank all alternatives. In Section 4, an example is given to illustrate the proposed MADM method. Finally, Section 5 provides concluding remarks and suggestions for future research.

\section{Preliminaries}

In this section, we introduce some basic knowledge and necessary concepts related to the IFS and IVIFS theories and the fuzzy cross entropy.

\subsection{Intuitionistic Fuzzy Sets (IFS) and Interval-Valued Intuitionistic Fuzzy Sets (IVIFS)}

Definition 1 (see [17]). Let $X=\left\{x_{1}, x_{2}, \ldots, x_{n}\right\}$ be a finite universal set. An IFS $A$ in $X$ can be expressed as $A=$ $\left\{\left\langle x_{i}, \mu_{A}\left(x_{i}\right), v_{A}\left(x_{i}\right)\right\rangle \mid x_{i} \in X\right\}$, where $\mu_{A}\left(x_{i}\right) \in[0,1], v_{A}\left(x_{i}\right) \in$ $[0,1]$, and $0 \leq \mu_{A}\left(x_{i}\right)+v_{A}\left(x_{i}\right) \leq 1$ for any $x_{i} \in X$. The numbers $\mu_{A}\left(x_{i}\right)$ and $v_{A}\left(x_{i}\right)$ denote the membership degree and nonmembership degree of the element $x_{i}$ in $A$, respectively.

If $A^{c}$ denotes the complement of $A$, then it is defined as follows:

$$
A^{c}=\left\{\left\langle x_{i}, v_{A}\left(x_{i}\right), \mu_{A}\left(x_{i}\right)\right\rangle \mid x_{i} \in X\right\} .
$$

Definition 2 (see [18]). Let $X=\left\{x_{1}, x_{2}, \ldots, x_{n}\right\}$ be a finite universal set. An IVIFS $\widetilde{A}$ in $X$ is defined as $\widetilde{A}=\left\{\left\langle x_{i}, \widetilde{\mu}_{\widetilde{A}}\left(x_{i}\right)\right.\right.$, $\left.\left.\widetilde{v}_{\widetilde{A}}\left(x_{i}\right)\right\rangle \mid x_{i} \in X\right\}=\left\{\left\langle x_{i},\left[\widetilde{\mu}_{\widetilde{A}}^{L}\left(x_{i}\right), \widetilde{\mu}_{\widetilde{A}}^{U}\left(x_{i}\right)\right],\left[\widetilde{v}_{\widetilde{A}}^{L}\left(x_{i}\right), \widetilde{v}_{\widetilde{A}}^{U}\left(x_{i}\right)\right]\right\rangle \mid\right.$ $\left.x_{i} \in X\right\}$, where $\widetilde{\mu}_{\widetilde{A}}\left(x_{i}\right) \in[0,1]$ and $\widetilde{v}_{\widetilde{A}}\left(x_{i}\right) \in[0,1]$ hold for every $x_{i} \in X$. The numbers $\tilde{\mu}_{A}\left(x_{i}\right)$ and $\widetilde{v}_{A}\left(x_{i}\right)$ denote the membership and nonmembership degrees of the element $x_{i}$ in $\widetilde{A}$, respectively. $\widetilde{\mu}_{\widetilde{A}}^{L}\left(x_{i}\right)$ and $\widetilde{\mu}_{\widetilde{A}}^{U}\left(x_{i}\right)$ express the lower and upper bounds of $\widetilde{\mu}_{\widetilde{A}}\left(x_{i}\right)$, respectively; $\widetilde{v}_{\widetilde{A}}^{L}\left(x_{i}\right)$ and $\widetilde{v}_{\widetilde{A}}^{U}\left(x_{i}\right)$ express the lower and upper bounds of $\widetilde{v}_{\widetilde{A}}\left(x_{i}\right)$, respectively, with the condition $0 \leq \widetilde{\mu}_{\widetilde{A}}^{U}\left(x_{i}\right)+\widetilde{v}_{\widetilde{A}}^{U}\left(x_{i}\right) \leq 1$.

If $\widetilde{A}^{c}$ denotes the complement of $\widetilde{A}$, then it is defined as follows:

$$
\begin{aligned}
\widetilde{A}^{c} & =\left\{\left\langle x_{i}, \widetilde{v}_{\widetilde{A}}\left(x_{i}\right), \widetilde{\mu}_{\widetilde{A}}\left(x_{i}\right)\right\rangle \mid x_{i} \in X\right\} \\
& =\left\{\left\langle x_{i},\left[\widetilde{v}_{\widetilde{A}}^{L}\left(x_{i}\right), \widetilde{v}_{\widetilde{A}}^{U}\left(x_{i}\right)\right],\left[\widetilde{\mu}_{\widetilde{A}}^{L}\left(x_{i}\right), \widetilde{\mu}_{\widetilde{A}}^{U}\left(x_{i}\right)\right]\right\rangle \mid x_{i} \in X\right\} .
\end{aligned}
$$

The expression IVIFS $(X)$ stands for all IVIFSs in $X[19]$.

Definition 3. $\tilde{a}=([a, b],[c, d])$ is an interval-valued intuitionistic fuzzy number (IVIFN) in IVIFS $A$, where $[a, b] \subseteq$ $[0,1]$ and $[c, d] \subseteq[0,1], b+d \leq 1 . \tilde{a}^{*}=([1,1],[0,0])$ is the biggest IVIFN. In particular, when $a=b$ and $c=d$, IVIFN $\tilde{a}$ degrades into the intuitionistic fuzzy number (IFN).

\subsection{IF Cross Entropy}

Definition 4 (see $[14,20]$ ). Let $A$ and $B$ be two IFSs in the universe $X=\left\{x_{1}, x_{2}, \ldots, x_{n}\right\}$, where $A=\left\{A\left(x_{1}\right), A\left(x_{2}\right), \ldots\right.$, $\left.A\left(x_{n}\right)\right\}$ and $B=\left\{B\left(x_{1}\right), B\left(x_{2}\right), \ldots, B\left(x_{n}\right)\right\}$. The IF cross entropy between $A$ and $B$ is defined as follows:

$$
\begin{aligned}
I_{\mathrm{IFS}}(A, B)=\sum_{i=1}^{n}[ & \mu_{A}\left(x_{i}\right) \log _{2} \frac{2 \mu_{A}\left(x_{i}\right)}{\mu_{A}\left(x_{i}\right)+\mu_{B}\left(x_{i}\right)} \\
& \left.+v_{A}\left(x_{i}\right) \log _{2} \frac{2 v_{A}\left(x_{i}\right)}{v_{A}\left(x_{i}\right)+v_{B}\left(x_{i}\right)}\right],
\end{aligned}
$$

where $I_{\mathrm{IFS}}(A, B)$ is used as the divergence measure between $A$ and $B$. In fact, $I_{\mathrm{IFS}}(A, B)$ can also be seen as a distance measure. However, $I_{\mathrm{IFS}}(A, B)$ is not symmetric. For this, a symmetric measure is defined as follows:

$$
D_{\mathrm{IFS}}(A, B)=I_{\mathrm{IFS}}(A, B)+I_{\mathrm{IFS}}(B, A),
$$

where $D_{\mathrm{IFS}}(A, B)$ is called the symmetric IF cross entropy between $A$ and $B$, or the discrimination degree, which is a symmetric discrimination information measure for IFS [17].

According to Shannon's inequality (see [21]), it can easily be verified that $D_{\mathrm{IFS}}(A, B) \geq 0, D_{\mathrm{IFS}}(A, B)=D_{\mathrm{IFS}}(B, A)$, $D_{\text {IFS }}(\widetilde{A}, \widetilde{B})=0$ iff $\widetilde{A}=\widetilde{B}$. Moreover, we can easily see that $D_{\mathrm{IFS}}\left(A^{c}, B^{c}\right)=D_{\mathrm{IFS}}(A, B)$, and Zhang and Jiang proved that $D_{\text {IFS }}\left(A, A^{c}\right)=2 n \log _{2} 2=2 n$, which is the maximum [20]; that is, $0 \leq D_{\mathrm{IFS}}(A, B) \leq 2 n$, and the maximum divergence measure between IVIFNs $a$ and $b$ is 2 . The larger the difference between $A$ and $B$ is, the larger $D_{\text {IFS }}(A, B)$ is and vice versa [16]. 
2.3. IVIF Cross Entropy. How to measure the distance between two IVIFNs is an important subject in the FS theory. Because the difference between the two systems can be defined by the cross entropy, the IVIF cross entropy is presented to define the information measure for the discrimination between two IVIFSs.

Definition 5. Similar to Zhang and Jiang [20] and Ye [15], we use the following definition of the IVIF cross entropy.

Let $\widetilde{A}$ and $\widetilde{B}$ be two IVIFSs in the universe $X=$ $\left\{x_{1}, x_{2}, \ldots, x_{n}\right\}$, where

$$
\begin{aligned}
\widetilde{A} & =\left\{\left\langle x_{i}, \widetilde{\mu}_{\widetilde{A}}\left(x_{i}\right), \widetilde{v}_{\widetilde{A}}\left(x_{i}\right)\right\rangle \mid x_{i} \in X\right\} \\
& =\left\{\left\langle x_{i},\left[\widetilde{\mu}_{\widetilde{A}}^{L}\left(x_{i}\right), \widetilde{\mu}_{\widetilde{A}}^{U}\left(x_{i}\right)\right],\left[\widetilde{v}_{\widetilde{A}}^{L}\left(x_{i}\right), \widetilde{v}_{\widetilde{A}}^{U}\left(x_{i}\right)\right]\right\rangle \mid x_{i} \in X\right\}, \\
\widetilde{B} & =\left\{\left\langle x_{i}, \widetilde{\mu}_{\widetilde{B}}\left(x_{i}\right), \widetilde{v}_{\widetilde{B}}\left(x_{i}\right)\right\rangle \mid x_{i} \in X\right\} \\
& =\left\{\left\langle x_{i},\left[\widetilde{\mu}_{\widetilde{B}}^{L}\left(x_{i}\right), \widetilde{\mu}_{\widetilde{B}}^{U}\left(x_{i}\right)\right],\left[\widetilde{v}_{\widetilde{A}}^{L}\left(x_{i}\right), \widetilde{v}_{\widetilde{A}}^{U}\left(x_{i}\right)\right]\right\rangle \mid x_{i} \in X\right\} .
\end{aligned}
$$

So, the average possible membership degree of element $x_{i}$ in IVIFSs $A$ and $B$ can be, respectively, defined as [20]

$$
\begin{aligned}
\bar{\mu}_{\widetilde{A}}\left(x_{i}\right) & =\frac{1}{2}\left(\frac{\tilde{\mu}_{\widetilde{A}}^{L}\left(x_{i}\right)+\tilde{\mu}_{\widetilde{A}}^{U}\left(x_{i}\right)}{2}+1-\frac{\widetilde{v}_{\widetilde{A}}^{L}\left(x_{i}\right)+\widetilde{v}_{\widetilde{A}}^{U}\left(x_{i}\right)}{2}\right) \\
& =\frac{\widetilde{\mu}_{\widetilde{A}}^{L}\left(x_{i}\right)+\widetilde{\mu}_{\widetilde{A}}^{U}\left(x_{i}\right)+2-\widetilde{v}_{\widetilde{A}}^{L}\left(x_{i}\right)-\widetilde{v}_{\widetilde{A}}^{U}\left(x_{i}\right)}{4},
\end{aligned}
$$$$
I_{\mathrm{IVIFS}}(\widetilde{A}, \widetilde{B})
$$$$
=\sum_{i=1}^{n} \frac{1}{4}\left(\tilde{\mu}_{\widetilde{A}}^{L}\left(x_{i}\right)+\widetilde{\mu}_{\widetilde{A}}^{U}\left(x_{i}\right)+2-\widetilde{v}_{\widetilde{A}}^{L}\left(x_{i}\right)-\widetilde{v}_{\widetilde{A}}^{U}\left(x_{i}\right)\right)
$$$$
\cdot \log _{2} \frac{\widetilde{\mu}_{\widetilde{A}}^{L}\left(x_{i}\right)+\widetilde{\mu}_{\widetilde{A}}^{U}\left(x_{i}\right)+2-\widetilde{v}_{\widetilde{A}}^{L}\left(x_{i}\right)-\widetilde{v}_{\widetilde{A}}^{U}\left(x_{i}\right)}{(1 / 2) \cdot\left[\left(\widetilde{\mu}_{\widetilde{A}}^{L}\left(x_{i}\right)+\widetilde{\mu}_{\widetilde{A}}^{U}\left(x_{i}\right)+2-\widetilde{v}_{\widetilde{A}}^{L}\left(x_{i}\right)-\widetilde{v}_{\widetilde{A}}^{U}\left(x_{i}\right)\right)+\left(\widetilde{\mu}_{\widetilde{B}}^{L}\left(x_{i}\right)+\widetilde{\mu}_{\widetilde{B}}^{U}\left(x_{i}\right)+2-\widetilde{v}_{\widetilde{B}}^{L}\left(x_{i}\right)-\widetilde{v}_{\widetilde{B}}^{U}\left(x_{i}\right)\right)\right]}
$$$$
+\sum_{i=1}^{n} \frac{1}{4}\left(2-\tilde{\mu}_{\widetilde{A}}^{L}\left(x_{i}\right)-\widetilde{\mu}_{\widetilde{A}}^{U}\left(x_{i}\right)+\widetilde{v}_{\widetilde{A}}^{L}\left(x_{i}\right)+\widetilde{v}_{\widetilde{A}}^{U}\left(x_{i}\right)\right)
$$$$
\cdot \log _{2} \frac{2-\widetilde{\mu}_{\widetilde{A}}^{L}\left(x_{i}\right)-\widetilde{\mu}_{\widetilde{A}}^{U}\left(x_{i}\right)+\widetilde{v}_{\widetilde{A}}^{L}\left(x_{i}\right)+\widetilde{v}_{\widetilde{A}}^{U}\left(x_{i}\right)}{(1 / 2) \cdot\left[\left(2-\widetilde{\mu}_{\widetilde{A}}^{L}\left(x_{i}\right)-\widetilde{\mu}_{\widetilde{A}}^{U}\left(x_{i}\right)+\widetilde{v}_{\widetilde{A}}^{L}\left(x_{i}\right)+\widetilde{v}_{\widetilde{A}}^{U}\left(x_{i}\right)\right)+\left(2-\widetilde{\mu}_{\widetilde{B}}^{L}\left(x_{i}\right)-\widetilde{\mu}_{\widetilde{B}}^{U}\left(x_{i}\right)+\widetilde{v}_{\widetilde{B}}^{L}\left(x_{i}\right)+\widetilde{v}_{\widetilde{B}}^{U}\left(x_{i}\right)\right)\right]},
$$

$$
\begin{aligned}
\bar{\mu}_{\widetilde{B}}\left(x_{i}\right) & =\frac{1}{2}\left(\frac{\widetilde{\mu}_{\widetilde{B}}^{L}\left(x_{i}\right)+\widetilde{\mu}_{\widetilde{B}}^{U}\left(x_{i}\right)}{2}+1-\frac{\widetilde{v}_{\widetilde{B}}^{L}\left(x_{i}\right)+\widetilde{v}_{\widetilde{B}}^{U}\left(x_{i}\right)}{2}\right) \\
& =\frac{\widetilde{\mu}_{\widetilde{B}}^{L}\left(x_{i}\right)+\widetilde{\mu}_{\widetilde{B}}^{U}\left(x_{i}\right)+2-\widetilde{v}_{\widetilde{B}}^{L}\left(x_{i}\right)-\widetilde{v}_{\widetilde{B}}^{U}\left(x_{i}\right)}{4} .
\end{aligned}
$$

The average possible nonmembership degree of element $x_{i}$ in IVIFSs $\widetilde{A}$ and $\widetilde{B}$ can be, respectively, defined as

$$
\begin{aligned}
\bar{v}_{\widetilde{A}}\left(x_{i}\right) & =1-\bar{\mu}_{\widetilde{A}}\left(x_{i}\right) \\
& =\frac{2-\widetilde{\mu}_{\widetilde{A}}^{L}\left(x_{i}\right)-\widetilde{\mu}_{\widetilde{A}}^{U}\left(x_{i}\right)+\widetilde{v}_{\widetilde{A}}^{L}\left(x_{i}\right)+\widetilde{v}_{\widetilde{A}}^{U}\left(x_{i}\right)}{4}, \\
\bar{v}_{\widetilde{B}}\left(x_{i}\right) & =1-\bar{\mu}_{\widetilde{B}}\left(x_{i}\right) \\
& =\frac{2-\widetilde{\mu}_{\widetilde{B}}^{L}\left(x_{i}\right)-\widetilde{\mu}_{\widetilde{B}}^{U}\left(x_{i}\right)+\widetilde{v}_{\widetilde{B}}^{L}\left(x_{i}\right)+\widetilde{v}_{\widetilde{B}}^{U}\left(x_{i}\right)}{4} .
\end{aligned}
$$

It is natural to define the fuzzy cross entropy between IVIFSs $\widetilde{A}$ and $\widetilde{B}$ as follows.

Definition 6 (see $[16,20])$. The IVIF cross entropy between IVIFSs $\widetilde{A}$ and $\widetilde{B}$ in the universe of discourse $X=$ $\left\{x_{1}, x_{2}, \ldots, x_{n}\right\}$ can be defined as where $I_{\mathrm{IVIFS}}(\widetilde{A}, \widetilde{B})$ is used to describe the discrimination measure between $\widetilde{A}$ and $\widetilde{B}$, which is also called the divergence measure between IVIFSs $\widetilde{A}$ and $\widetilde{B}[22]$. In fact, $I_{\text {IVIFS }}(\widetilde{A}, \widetilde{B})$ can also be seen as the distance measure. However, $I_{\text {IVIFS }}(\widetilde{A}, \widetilde{B})$ is not symmetric. For this, it should be modified to the symmetric fuzzy cross entropy as follows:

$$
D_{\mathrm{IVIFS}}(\widetilde{A}, \widetilde{B})=I_{\mathrm{IVIFS}}(\widetilde{A}, \widetilde{B})+I_{\mathrm{IVIFS}}(\widetilde{B}, \widetilde{A}),
$$


vice versa. Additionally, it is immediate that $D_{\mathrm{IVIFS}}(\widetilde{A}, \widetilde{B})=$ $D_{\text {IVIFS }}\left(\widetilde{A}^{c}, \widetilde{B}^{c}\right)$, and $D_{\text {IVIFS }}(\widetilde{A}, \widetilde{B})=D_{\text {IVIFS }}(\widetilde{B}, \widetilde{A})$.

Usually, the weight of the element $x_{i} \in X$ should be taken into account, so the IVIF weighted cross entropy and weighted discrimination degree of $\widetilde{A}$ from $\widetilde{B}$ can be defined as follows.

Definition 7. Assume the weight of the element $x_{i} \in X$ is $w_{i}$, where $\sum_{i=1}^{n} w_{i}=1$. For two IVIFSs $\widetilde{A}$ and $\widetilde{B}$, let the interval-valued intuitionistic fuzzy cross-entropy weighted averaging (IVIFIWA) operator be expressed as

$$
\operatorname{IVIFIWA}_{w}(\widetilde{A}, \widetilde{B})=\sum_{i=1}^{n}\left(w_{i} I_{\mathrm{IVIFS}}\left(\widetilde{A}\left(x_{i}\right), \widetilde{B}\left(x_{i}\right)\right)\right) .
$$

In particular, if $w_{i}=1 / n$, the IVIFIWA operator is reduced to the IVIFIA operator.

So, using the IVIFIWA operator, $I_{w \mathrm{IVIFS}}(\widetilde{A}, \widetilde{B})$, as the IVIF weighted cross entropy between $\widetilde{A}$ and $\widetilde{B}$ can be expressed as follows:

$$
\begin{aligned}
& I_{w \mathrm{IVIFS}}(\widetilde{A}, \widetilde{B}) \\
& =\sum_{i=1}^{n} w_{i}\left(\frac{1}{4}\left(\widetilde{\mu}_{\widetilde{A}}^{L}\left(x_{i}\right)+\widetilde{\mu}_{\widetilde{A}}^{U}\left(x_{i}\right)+2-\widetilde{v}_{\widetilde{A}}^{L}\left(x_{i}\right)-\widetilde{v}_{\widetilde{A}}^{U}\left(x_{i}\right)\right)\right. \\
& \left.\cdot \log _{2} \frac{\widetilde{\mu}_{\widetilde{A}}^{L}\left(x_{i}\right)+\widetilde{\mu}_{\widetilde{A}}^{U}\left(x_{i}\right)+2-\widetilde{v}_{\widetilde{A}}^{L}\left(x_{i}\right)-\widetilde{v}_{\widetilde{A}}^{U}\left(x_{i}\right)}{(1 / 2) \cdot\left[\left(\widetilde{\mu}_{\widetilde{A}}^{L}\left(x_{i}\right)+\widetilde{\mu}_{\widetilde{A}}^{U}\left(x_{i}\right)+2-\widetilde{v}_{\widetilde{A}}^{L}\left(x_{i}\right)-\widetilde{v}_{\widetilde{A}}^{U}\left(x_{i}\right)\right)+\left(\widetilde{\mu}_{\widetilde{B}}^{L}\left(x_{i}\right)+\widetilde{\mu}_{\widetilde{B}}^{U}\left(x_{i}\right)+2-\widetilde{v}_{\widetilde{B}}^{L}\left(x_{i}\right)-\widetilde{v}_{\widetilde{B}}^{U}\left(x_{i}\right)\right)\right]}\right) \\
& +\sum_{i=1}^{n} w_{i}\left(\frac{1}{4}\left(2-\widetilde{\mu}_{\widetilde{A}}^{L}\left(x_{i}\right)-\widetilde{\mu}_{\widetilde{A}}^{U}\left(x_{i}\right)+\widetilde{v}_{\widetilde{A}}^{L}\left(x_{i}\right)+\widetilde{v}_{\widetilde{A}}^{U}\left(x_{i}\right)\right)\right. \\
& \left.\cdot \log _{2} \frac{2-\widetilde{\mu}_{\widetilde{A}}^{L}\left(x_{i}\right)-\widetilde{\mu}_{\widetilde{A}}^{U}\left(x_{i}\right)+\widetilde{v}_{\widetilde{A}}^{L}\left(x_{i}\right)+\widetilde{v}_{\widetilde{A}}^{U}\left(x_{i}\right)}{(1 / 2) \cdot\left[\left(2-\widetilde{\mu}_{\widetilde{A}}^{L}\left(x_{i}\right)-\widetilde{\mu}_{\widetilde{A}}^{U}\left(x_{i}\right)+\widetilde{v}_{\widetilde{A}}^{L}\left(x_{i}\right)+\widetilde{v}_{\widetilde{A}}^{U}\left(x_{i}\right)\right)+\left(2-\widetilde{\mu}_{\widetilde{B}}^{L}\left(x_{i}\right)-\widetilde{\mu}_{\widetilde{B}}^{U}\left(x_{i}\right)+\widetilde{v}_{\widetilde{B}}^{L}\left(x_{i}\right)+\widetilde{v}_{\widetilde{B}}^{U}\left(x_{i}\right)\right)\right]}\right) .
\end{aligned}
$$

Similarly, the interval-valued intuitionistic fuzzy discrimination degree weighted averaging (IVIFDWA) operator is expressed as

$$
\operatorname{IVIFWDA}_{w}(\widetilde{A}, \widetilde{B})=\sum_{i=1}^{n} w_{i} \cdot D_{\mathrm{IVIFS}}\left(\widetilde{A}\left(x_{i}\right), \widetilde{B}\left(x_{i}\right)\right) .
$$

\section{The Novel Approach to MADM Based on IVIF Cross Entropy}

This section introduces a novel approach to the MADM problem for determining completely unknown attribute weights using a nonlinear programming model and for ranking all alternatives based on the weighted symmetric fuzzy cross entropy (the weighted discrimination degree) and the idea of using TOPSIS in the IVIF environment.

The main procedure of the decision method proposed is presented below.

Step 1 (constitute an interval-valued intuitionistic fuzzy decision matrix $\left.\widetilde{R}_{i j}\right)$. Assume that $\left\{P_{i} \mid i=1,2, \ldots, m\right\}$ is the set of alternatives and $\left\{Q_{j} \mid j=1,2, \ldots, n\right\}$ is the set of attributes. Different evaluating values $r_{i j}^{k}$ of $i$ th alternative $P_{i}$ with respect to $j$ th attribute $Q_{j}$ are provided by the decision maker based on IVIFNs, in order to constitute the intervalvalued intuitionistic fuzzy decision matrix $\widetilde{R}_{i j}=\left(\widetilde{r}_{i j}\right)_{m \times n}$ :

$$
\widetilde{R}_{i j}=\left(\widetilde{r}_{i j}\right)_{m \times n}=\left(\left[\widetilde{\mu}_{i j}^{L}, \widetilde{\mu}_{i j}^{U}\right],\left[\widetilde{v}_{i j}^{L}, \widetilde{v}_{i j}^{U}\right]\right)_{m \times n} .
$$

Step 2 (determine the PIS $P^{+}$and the NIS $P^{-}$). TOPSIS is a method to solve MADM problems, which suggests to choose the alternative with the shortest distance from the PIS and the farthest distance from the NIS [17].

According to [23], under the IVIF environment, the PIS and NIS, respectively denoted as $P^{+}$and $P^{-}$, are represented as follows:

$$
\begin{aligned}
P^{+} & =\left\{\left\langle Q_{j}, r_{j}^{+}\left(Q_{j}\right)\right\rangle \mid Q_{j} \in Q\right\} \\
& =\left\{\left\langle Q_{j},([1,1],[0,0])\right\rangle \mid Q_{j} \in Q\right\}, \\
P^{-} & =\left\{\left\langle Q_{j}, r_{j}^{-}\left(Q_{j}\right)\right\rangle \mid Q_{j} \in Q\right\} \\
& =\left\{\left\langle Q_{j},([0,0],[1,1])\right\rangle \mid Q_{j} \in Q\right\} .
\end{aligned}
$$

Step 3 (calculate the discrimination degree between alternatives $P_{i}$ and $P^{+}\left(P^{-}\right)$, which is expressed by $D_{i j}^{+}\left(D_{i j}^{-}\right)$). Consider

$$
D_{i j}^{+}=\left[D\left(r_{i j}, r_{j}^{+}\right)\right]_{m \times n}, \quad D_{i j}^{-}=\left[D\left(r_{i j}, r_{j}^{-}\right)\right]_{m \times n},
$$

where $D\left(r_{i j}, r_{j}^{+}\right)\left(D\left(r_{i j}, r_{j}^{-}\right)\right)$denotes the discrimination degree between alternatives $P_{i}$ and $P^{+}\left(P^{-}\right)$with respect to $j$ th attribute $Q_{j}$. Also,

$$
\begin{aligned}
& D\left(r_{i j}, r_{j}^{+}\right)=I_{\text {IVIFNs }}\left(r_{i j}, r_{j}^{+}\right)+I_{\text {IVIFNs }}\left(r_{j}^{+}, r_{i j}\right), \\
& D\left(r_{i j}, r_{j}^{-}\right)=I_{\text {IVIFNs }}\left(r_{i j}, r_{j}^{-}\right)+I_{\text {IVIFNs }}\left(r_{i j}, r_{j}^{-}\right) .
\end{aligned}
$$


Step 4 (determine the weight vector of attributes $\omega^{+}$and $\omega^{-}$ with PIS and NIS as reference objects, resp.).

Modeling Principle. Let $\omega^{+}=\left(\omega_{1}^{+}, \omega_{2}^{+}, \ldots, \omega_{n}^{+}\right)$be the weight vector of attributes with PIS as the reference object, where $\omega_{j}^{+} \geq 0, j=1,2, \ldots, n$, and $\sum_{j=1}^{n} \omega_{j}^{+}=1$. Similarly, let $\omega^{-}=$ $\left(\omega_{1}^{-}, \omega_{2}^{-}, \ldots, \omega_{n}^{-}\right)$be the weight vector of attributes with NIS as the reference object, where $\omega_{j}^{-} \geq 0, j=1,2, \ldots, n$, and $\sum_{j=1}^{n} \omega_{j}^{-}=1$.

Generally, the smaller the discrimination degree between alternatives $P_{i}$ and $P^{+}$is, that is, $D\left(r_{i j}, r_{j}^{+}\right) \rightarrow 0$, the better the $P_{i}$ is; the larger the discrimination degree between alternatives $P_{i}$ and $P^{-}$is, that is, $D\left(r_{i j}, r_{j}^{-}\right) \rightarrow 2$, the better the $P_{i}$ is. According to this, considering the weight of each attribute, the deviation of the discrimination degree between alternatives $P_{i}$ and $P^{+}$with respect to the $j$ th attribute $Q_{j}$ is defined as $D\left(r_{i j}, r_{j}^{+}\right)-0$. The measure of the total deviation of the discrimination degrees usually takes the form of the sum of squares. So, the sum of weighted deviations between alternatives $P_{i}$ and $P^{+}$with respect to all the attributes is $\sum_{j=1}^{n}\left[\omega_{j}^{+}\left(D\left(r_{i j}, r_{j}^{+}\right)-0\right)\right]^{2}=\sum_{i=1}^{m} \sum_{j=1}^{n}\left[\omega_{j}^{+} D\left(r_{i j}, r_{j}^{+}\right)\right]^{2}$. We will minimize the sum of weighted deviations in order to determine the weight vector. To solve for the weights $\omega_{j}^{+}$, the following nonlinear programming model can be established:

$$
\begin{array}{ll}
\min & Z\left(w^{+}\right)=\sum_{i=1}^{m} \sum_{j=1}^{n}\left[\omega_{j}^{+} D\left(r_{i j}, r_{j}^{+}\right)\right]^{2} \\
\text { st. } & \left\{\begin{array}{l}
\sum_{j=1}^{n} \omega_{j}^{+}=1 \\
\omega_{j}^{+} \geq 0, \quad j=1,2, \ldots, n .
\end{array}\right.
\end{array}
$$

Using the Lagrange multiplier conditional extreme value method, we obtain the following Lagrangian function:

$$
L\left(w^{+}, \lambda\right)=\sum_{i=1}^{m} \sum_{j=1}^{n}\left[\omega_{j}^{+} D\left(r_{i j}, r_{j}^{+}\right)\right]^{2}+2 \lambda\left(\sum_{j=1}^{n} \omega_{j}^{+}-1\right),
$$

where $\lambda$ is the Lagrange multiplier. To obtain the weights $\omega_{j}^{+}$, we differentiate (18), $d f\left(L\left(w^{+}, \lambda\right)\right) / d w^{+}=0$ and $d f\left(L\left(w^{+}, \lambda\right)\right) / d \lambda=0$ and obtain the following result:

$$
\begin{gathered}
\omega_{j}^{+} \sum_{i=1}^{m} D\left(r_{i j}, r_{j}^{+}\right)^{2}+\lambda=0, \\
\sum_{j=1}^{n} \omega_{j}^{+}-1=0 .
\end{gathered}
$$
$\omega_{j}^{+}$:

By solving the system, we can obtain the unique weights

$$
\omega_{j}^{+}=\frac{\left[\sum_{j=1}^{n}\left(\sum_{i=1}^{m} D\left(r_{i j}, r_{j}^{+}\right)^{2}\right)^{-1}\right]^{-1}}{\sum_{i=1}^{m} D\left(r_{i j}, r_{j}^{+}\right)^{2}} .
$$

Similarly, according to the idea above, considering the weight of each attribute, the deviation of the discrimination degree between alternatives $P_{i}$ and $P^{-}$with respect to the $j$ th attribute $Q_{j}$ is defined as $D\left(r_{i j}, r_{j}^{-}\right)-2$. The total deviation of the discrimination degrees is usually measured as the sum of squares. So, the sum of weighted deviation degrees between alternatives $P_{i}$ and $P^{-}$with respect to all the attributes is $\sum_{j=1}^{n}\left[\omega_{j}^{+}\left(D\left(r_{i j}, r_{j}^{+}\right)-2\right)\right]^{2}$. It should be minimized in order to determine the weight vector. To solve for the weights $\omega_{j}^{+}$, the following nonlinear programming model can be established:

$$
\begin{array}{ll}
\min & Z\left(w^{+}\right)=\sum_{i=1}^{m} \sum_{j=1}^{n}\left[\omega_{j}^{-} D\left(r_{i j}, r_{j}^{-}-2\right)\right]^{2} \\
\text { st. } \quad & \left\{\begin{array}{l}
\sum_{j=1}^{n} \omega_{j}^{-}=1 \\
\omega_{j}^{+} \geq 0, \quad j=1,2, \ldots, n .
\end{array}\right.
\end{array}
$$

A similar procedure can be easily adapted to obtain the unique weights $\omega_{j}^{-}$:

$$
\omega_{j}^{-}=\frac{\left[\sum_{j=1}^{n}\left(\sum_{i=1}^{m}\left(D\left(r_{i j}, r_{j}^{-}\right)-2\right)^{2}\right)^{-1}\right]^{-1}}{\sum_{i=1}^{m}\left(D\left(r_{i j}, r_{j}^{-}\right)-2\right)^{2}} .
$$

Step 5 (use the IVIFWDA operator to aggregate $\omega^{+}$and $D_{i j}^{+}$ $\left(\omega^{-}\right.$and $\left.\left.D_{i j}^{-}\right)\right)$. The weighted discrimination degrees $G^{+}\left(G^{-}\right)$ between $P_{i}$ and $P^{+}\left(P^{-}\right)$can be acquired as follows:

$$
\begin{aligned}
& G^{+}=\left(G_{1}^{+}, G_{2}^{+}, \ldots, G_{m}^{+}\right), \\
& \text {where } G_{i}^{+}=\sum_{j=1}^{n} w_{j}^{+} \times D\left(r_{i j}, r_{j}^{+}\right) \\
& G^{-}=\left(G_{1}^{-}, G_{2}^{-}, \ldots, G_{m}^{-}\right), \\
& \text {where } G_{i}^{-}=\sum_{j=1}^{n} w_{j}^{-} \times D\left(r_{i j}, r_{j}^{-}\right) .
\end{aligned}
$$

Step 6 (rank all alternatives). In general, based on the TOPSIS, a relative closeness coefficient is defined to determine the ranking order of all alternatives. In the paper, we order all alternatives according to the relative closeness coefficient $\left(g_{i}\right)$ obtained using the extended TOPSIS. The equation for the relative closeness coefficient is as follows:

$$
g_{i}=\frac{G_{i}^{+}}{G_{i}^{+}+G_{i}^{-}} \text {. }
$$

The smaller $g_{i}$, the better the alternative $P_{i}$.

\section{Some Examples to Illustrate the Approach}

Recently, the IVIFS, as a useful tool to deal with imperfect facts and data, as well as imprecise knowledge, has been used to solve MADM problems related to the field of engineering [24]. So, in this section, we provide two examples of MADM problems in the engineering to demonstrate the application of the proposed method, as well as its effectiveness. Example 1 about the evaluation of the security of a wireless sensor network (WSN) and Example 2 about the problem of choosing the best suppliers are described below. 


\subsection{Description and Decision Model}

Example 1. WSN, as an integrated network which can perform information gathering, processing, and delivering, can connect the real and logistic information worlds. It greatly changes the interaction between people and the nature. There are many potential applications for WSN, such as in the industry, agriculture, military affairs, environment monitoring, biomedicine, city management, and disaster emergencies. The data privacy protection is essential for widespread deployment of WSN. However, the characteristics of a WSN make it a serious challenge, which attracted more attention recently [24].

The data privacy protection protocols in WSN tend to aim at a particular data operation. Only a few specific data operations are supported; therefore, the operation types supported by the protocols must be taken into account. Due to the WSN resource restrictions and the demand from applications for the precision of the queried and gathered data, the algorithm performance and outcome precision should be considered as well. Assume there is a certain corporation plan to pick the best WSN among five potential candidates $P_{i}(i=1,2,3,4,5)$ from the perspective of the data privacy protection ability. Based on the above, there are five attributes $Q_{j}(j=1,2,3,4,5)$ to evaluate alternatives: $Q_{1}$ is the operation types supported; $Q_{2}$ is the precision of gathering and querying; $Q_{3}$ is the privacy protection ability; $Q_{4}$ is the algorithm performance; $Q_{5}$ is the outcome precision. The decision maker provides the evaluation information on alternatives with regard to attributes using IVIFNs in the following decision-making matrix $\widetilde{R}_{i j}$ :

$$
\begin{aligned}
& \widetilde{R}_{i j} \\
& =\left(\begin{array}{cccccc}
P_{1} & ([0.4,0.5],[0.32,0.4]) & ([0.67,0.78],[0.14,0.2]) & ([0.5,0.65],[0.13,0.22]) & ([0.45,0.6],[0.3,0.35]) & ([0.6,0.65],[0.18,0.3]) \\
P_{2} & ([0.52,0.6],[0.1,0.17]) & ([0.56,0.68],[0.23,0.28]) & ([0.65,0.7],[0.2,0.25]) & ([0.56,0.62],[0.2,0.28]) & ([0.55,0.68],[0.15,0.28]) \\
P_{3} & ([0.62,0.72],[0.2,0.25]) & ([0.35,0.45],[0.33,0.43]) & ([0.55,0.63],[0.28,0.32]) & ([0.45,0.62],[0.19,0.3]) & ([0.63,0.67],[0.16,0.2]) \\
P_{4} & ([0.4,0.48],[0.4,0.5]) & ([0.4,0.5],[0.2,0.5]) & ([0.5,0.8],[0.1,0.2]) & ([0.55,0.75],[0.15,0.25]) & ([0.45,0.65],[0.25,0.35]) \\
P_{5} & ([0.4,0.5],[0.4,0.5]) & ([0.3,0.6],[0.3,0.4]) & ([0.6,0.7],[0.05,0.25]) & ([0.6,0.7],[0.1,0.3]) & ([0.5,0.6],[0.2,0.4])
\end{array}\right) .
\end{aligned}
$$

We then calculate the discrimination degree between each alternative and $P^{+}\left(P^{-}\right)$:

$$
\begin{aligned}
& D\left(r_{i j}, r_{j}^{+}\right) \\
& =\left(\begin{array}{cccccc} 
& Q_{1} & Q_{2} & Q_{3} & Q_{4} & Q_{5} \\
P_{1} & 0.5531 & 0.2426 & 0.3384 & 0.4729 & 0.3480 \\
P_{2} & 0.3225 & 0.3610 & 0.3068 & 0.3708 & 0.3384 \\
P_{3} & 0.3099 & 0.6068 & 0.4107 & 0.4107 & 0.2943 \\
P_{4} & 0.6305 & 0.5456 & 0.2912 & 0.3225 & 0.4209 \\
P_{5} & 0.6226 & 0.5456 & 0.2759 & 0.3068 & 0.4380
\end{array}\right), \\
& D\left(r_{i j}, r_{j}^{-}\right) \\
&
\end{aligned}
$$

Applying (20) and (22), the vector of weights $\omega^{+}\left(\omega^{-}\right)$of $P_{i}(i=1,2, \ldots, 5)$ is as follows:

$$
\begin{aligned}
\omega^{+} & =\left(\omega_{1}^{+}, \omega_{2}^{+}, \ldots, \omega_{5}^{+}\right) \\
& =(0.1225,0.1372,0.2940,0.2174,0.2289), \\
\omega^{-} & =\left(\omega_{1}^{-}, \omega_{2}^{-}, \ldots, \omega_{5}^{-}\right) \\
& =(0.1616,0.1710,0.2452,0.2085,0.2137) .
\end{aligned}
$$

Applying (23), the weighted arithmetic degree of discrimination is as follows:

$$
\begin{aligned}
G^{+} & =\left(G_{1}^{+}, G_{2}^{+}, \ldots, G_{5}^{+}\right) \\
& =(0.3830,0.3373,0.3986,0.4042,0.3992), \\
G^{-} & =\left(G_{1}^{-}, G_{2}^{-}, \ldots, G_{5}^{-}\right) \\
& =(0.9259,0.9874,0.9010,0.8773,0.88620) .
\end{aligned}
$$

Finally, applying (24), we obtain $g_{1}=0.2926, g_{2}=$ $0.2546, g_{3}=0.3067, g_{4}=0.3154$, and $g_{5}=0.3106$. According to the values of $g_{i}$, the ranking order of the five alternatives is $P_{2}>P_{1}>P_{3}>P_{5}>P_{4}$, and thus the most desirable alternative is $P_{2}$.

Example 2. In the supply chain environment, more enterprises have recently started paying attention to the selection of suppliers. It is important for the supply chain management to reasonably select suppliers in a scientific way. A famous nuclear power equipment manufacturing enterprise desires selecting the most appropriate supplier for one of its most critical parts used in its assembling process. In order to solve the problem, after the preevaluation, four suppliers $P_{i}(i=$ $1,2,3,4)$ have been selected as alternatives for further evaluation. To evaluate alternative suppliers, the enterprise must make a decision according to the following four attributes: $Q_{1}$ is the product quality, $Q_{2}$ is the relationship closeness, $Q_{3}$ is the delivery performance, $Q_{4}$ is the overall cost of the product, where $Q_{1}, Q_{2}$ and $Q_{3}$ are the benefit attributes, and $Q_{4}$ is the cost attribute. The decision maker provides the evaluation information on alternatives with regard to 
attributes using IVIFNs as the following decision-making matrix $\widetilde{R}_{i j}$ :

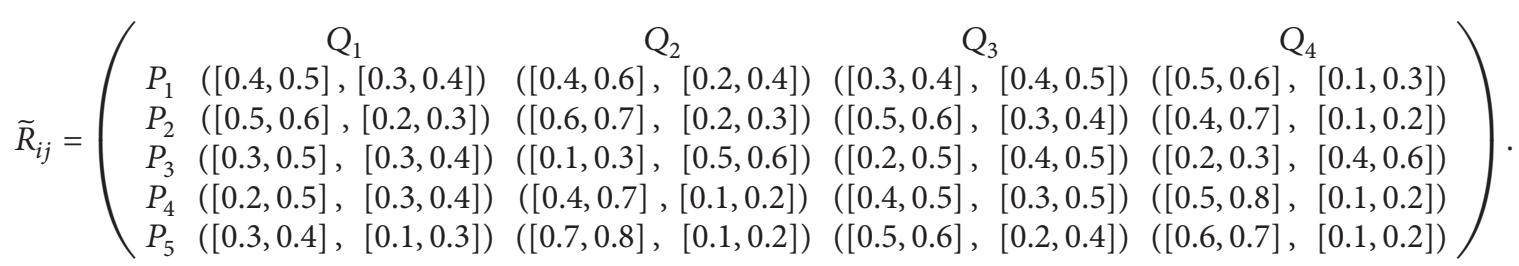

We then calculate the degree of discrimination between alternatives and $P^{+}\left(P^{-}\right)$:

$$
\begin{gathered}
D\left(r_{i j}, r_{j}^{+}\right) \\
=\left(\begin{array}{ccccc} 
& Q_{1} & Q_{2} & Q_{3} & Q_{4} \\
P_{1} & 0.5456 & 0.4729 & 0.7043 & 0.3708 \\
P_{2} & 0.4040 & 0.3384 & 0.4729 & 0.3384 \\
P_{3} & 0.5835 & 0.9351 & 0.7043 & 0.8376 \\
P_{4} & 0.6226 & 0.3384 & 0.5835 & 0.2759 \\
P_{5} & 0.5088 & 0.2161 & 0.4380 & 0.2759
\end{array}\right), \\
D\left(r_{i j}, r_{j}^{-}\right) \\
\end{gathered}
$$

Applying (20) and (22), the vector of weights $\omega^{+}\left(\omega^{-}\right)$of $P_{i}(i=1,2, \ldots, 5)$ is as follows:

$$
\begin{aligned}
\omega^{+} & =\left(\omega_{1}^{+}, \omega_{2}^{+}, \ldots, \omega_{n}^{+}\right) \\
& =(0.2386,0.2515,0.1976,0.3124), \\
\omega^{-} & =\left(\omega_{1}^{-}, \omega_{2}^{-}, \ldots, \omega_{n}^{-}\right) \\
& =(0.2250,0.2714,0.2083,0.2953) .
\end{aligned}
$$

Applying (23), the weighted arithmetic degree of discrimination is as follows:

$$
\begin{aligned}
G^{+} & =\left(G_{1}^{+}, G_{2}^{+}, \ldots, G_{m}^{+}\right) \\
& =(0.5041,0.3806,0.7752,0.4351,0.3484), \\
G^{-} & =\left(G_{1}^{-}, G_{2}^{-}, \ldots, G_{m}^{-}\right) \\
& =(0.7631,0.9234,0.4927,0.8701,0.9978) .
\end{aligned}
$$

Finally, applying (24), we obtain $g_{1}=0.4117, g_{2}=$ $0.2987, g_{3}=0.6055, g_{4}=0.3494$, and $g_{5}=0.2698$. According to the values of $g_{i}$, the ranking order of the five alternatives is $P_{5}>P_{2}>P_{4}>P_{1}>P_{3}$, and thus the most desirable alternative is $P_{5}$.
4.2. Comparative Analysis and Discussion. In this subsection, the comparative analysis is conducted in order to validate the results of the proposed approach and compare it to other approaches and to illustrate the most important characteristics of our approach.

To conduct the comparative analysis, we apply the methods described in [25-27] to the same decision-making problems mentioned in Section 4.1. According to all the methods, the ranking order of Example 1 is $P_{2}>P_{1}>P_{3}>P_{5}>P_{4}$, and the most desirable alternative is $P_{2}$; the ranking order of Example 2 is $P_{5}>P_{2}>P_{4}>P_{1}>P_{3}$, and the most desirable alternative is $P_{5}$. These ordering results are in accordance with the results obtained by the proposed method in the paper. From the results, the proposed decision-making method in this paper can be suitably utilized to solve the IVIF MADM problems with completely unknown attribute weights.

Although the decision results obtained by the approaches in [25-27] are the same as using our proposed method, the decision principles and processes are remarkably different. First, with respect to the determination of the completely unknown attribute weights, the methods in [25-27] all use the IVIF entropy. In our paper, the completely unknown attribute weights are obtained using the IVIF cross entropy. The former simply takes into account the ambiguity of the evaluation information itself to determine attribute weights. The latter takes into consideration the differences between the evaluation information and IVIF PIS, as well as IVIF NIS. This can be seen as the information measure of the discrimination between two IVIFSs, used to determine the attribute weight vectors $\omega^{+}$and $\omega^{-}$with PIS and NIS as the reference object, respectively, using the IVIF cross entropy. Second, with respect to the ranking of all alternatives, in [25], all alternatives are ranked using the weighted correlation coefficients; in [26], all alternatives are ranked based on the TOPSIS by using the weighted distance measure; and, in [27], the authors calculated the weighted arithmetic average for each alternative using the IVIF weighted arithmetic average operator and then ranked all alternatives according to the score function. However, the proposed approach in the paper is to rank all alternatives directly using the fuzzy cross entropy of IVIFSs. From this point of view, using any approach described in [25-27] makes the process of decision making more timeconsuming. Besides, Wang et al. [28] and Ye [29] pointed out that the score function in [27] has some limitations under certain conditions; for example, the isotonicity of the alternatives does not hold. These drawbacks of the approaches proposed in [25-27] make our approach more suitable in practice. 
In addition, the information about attribute weights in most papers is usually completely known [30-33] or incompletely known [23, 29, 34, 35] because of the time pressure, lack of knowledge or data, and expert's limited expertise in the problem domain. However, when the information about the weights of the attributes is completely unknown, the methods described above will not work well. In our paper, we propose a method to determine the attributes' weights. Thus, our method can be considered to be complement to the methods in $[23,29-35]$.

To sum up, the proposed method has the following characteristics. First, since the proposed approach aims to minimize the total deviation of the discrimination degree between each alternative and the PIS (NIS), it is intuitive and easy to understand. Second, we propose a novel approach to obtain completely unknown attribute weights with PIS (NIS) as the reference object using the IVIF cross entropy. Finally, the proposed approach has clear concept and simple calculations and provides a new idea for solving decisionmaking problems under the IVIF environment, so it can be easily used in many areas for practical decision problems that involve choosing an optimal alternative from a list of alternatives when multiple attributes must be considered.

\section{Conclusion}

The IVIFS and entropy theories, as very important notions in the fuzzy set theory, have drawn the attention of many researchers to deal with MADM problems in different fields. However, there is little research directly using the IVIF cross entropy to determine the attribute weights and rank alternatives. Attribute weights perform an important function in MADM problems, for the variation in attribute weights may lead to different ranking orders of alternatives. We present a novel MADM approach for the problem with completely unknown attribute weights in the framework of IVIFS. First, the fuzzy cross entropy and discrimination degree of IVIFS are defined. Subsequently, based on the discrimination degree of IVIFS, a nonlinear programming model to minimize the total deviation of discrimination degrees between alternatives and the PIS (NIS) is constructed to obtain the attribute weights and, then, the weighted discrimination degree. Finally, all the alternatives are ranked according to the relative closeness coefficients using the extended TOPSIS method, and the most desirable alternative is chosen. We illustrate the feasibility and validity of the proposed method by two examples.

The paper extends the theory and methodology of the MADM analysis using the IVIF cross entropy. The main advantage of the proposed method is its ability to handle the decision-making problems in which the ratings of alternatives with respect to completely unknown attribute weights are represented by IVIFSs. At the same time, the method proposed in the paper can be used to solve MADM problems with completely unknown attribute weights under IF environments, as well as with complete weight information under IF or IVIF environments. In addition, we present a comparative study with some existing decision-making methods. Compared to the existing methods, our proposed approach has characteristics and advantages and can provide a useful technique to efficiently assist the DMs. The plan of our future work is to apply the proposed method to some other practical decision-making problems, such as the performance evaluation, water resource schedule, and risk investment.

\section{Conflict of Interests}

The authors declare that there is no conflict of interests regarding the publication of this paper.

\section{Acknowledgments}

The authors wish to thank the Editor G.M. Xie and anonymous reviewers for the valuable comments and suggestions which were very helpful for improvement of the paper. The work is supported by the Shanghai Committee of Science and Technology, China (Grant no. 13692107200).

\section{References}

[1] L. A. Zadeh, "Fuzzy sets," Information and Computation, vol. 8, pp. 338-353, 1965.

[2] K. T. Atanassov, "Intuitionistic fuzzy sets," Fuzzy Sets and Systems, vol. 20, no. 1, pp. 87-96, 1986.

[3] K. Atanassov and G. Gargov, "Interval valued intuitionistic fuzzy sets," Fuzzy Sets and Systems, vol. 31, no. 3, pp. 343-349, 1989.

[4] Z. S. Xu and R. R. Yager, "Intuitionistic and interval-valued intuitionistic fuzzy preference relations and their measures of similarity for the evaluation of agreement within a group," Fuzzy Optimization and Decision Making, vol. 8, no. 2, pp. 123-139, 2009.

[5] K. Atanassov and C. Georgiev, "Intuitionistic fuzzy Prolog," Fuzzy Sets and Systems, vol. 53, no. 2, pp. 121-128, 1993.

[6] V. Khatibi and G. A. Montazer, "Intuitionistic fuzzy set vs. fuzzy set application in medical pattern recognition," Artificial Intelligence in Medicine, vol. 47, no. 1, pp. 43-52, 2009.

[7] E. Straszecka, "Combining uncertainty and imprecision in models of medical diagnosis," Information Sciences, vol. 176, no. 20, pp. 3026-3059, 2006.

[8] W.-L. Hung and M.-S. Yang, "On the J-divergence of intuitionistic fuzzy sets with its application to pattern recognition," Information Sciences, vol. 178, no. 6, pp. 1641-1650, 2008.

[9] L. A. Zadeh, "Probability measures of fuzzy events," Journal of Mathematical Analysis and Applications, vol. 23, pp. 421-427, 1968.

[10] P. Burillo and H. Bustince, "Entropy on intuitionistic fuzzy sets and on interval-valued fuzzy sets," Fuzzy Sets and Systems, vol. 78, no. 3, pp. 305-316, 1996.

[11] C. E. Shannon, "A mathematical theory of communication," The Bell System Technical Journal, vol. 27, pp. 379-423, 1948.

[12] S. Kullback and R. A. Leibler, "On information and sufficiency," The Annals of Mathematical Statistics, vol. 22, no. 1, pp. 79-86, 1951.

[13] X.-G. Shang and W.-S. Jiang, "A note on fuzzy information measures," Pattern Recognition Letters, vol. 18, no. 5, pp. 425432, 1997. 
[14] I. K. Vlachos and G. D. Sergiadis, "Intuitionistic fuzzy information-applications to pattern recognition," Pattern Recognition Letters, vol. 28, no. 2, pp. 197-206, 2007.

[15] J. Ye, "Multicriteria fuzzy decision-making method based on the intuitionistic fuzzy cross-entropy," in Proceedings of the International Conference on Intelligent Human-Machine Systems and Cybernetics (IHMSC '09), Y. C. Tang, J. Lawry, and V. N. Huynh, Eds., vol. 1, pp. 59-61, Hangzhou, China, August 2009.

[16] J. Ye, "Fuzzy cross entropy of interval-valued intuitionistic fuzzy sets and its optimal decision-making method based on the weights of alternatives," Expert Systems with Applications, vol. 38, no. 5, pp. 6179-6183, 2011.

[17] H. M. Zhang and L. Y. Yu, "MADM method based on crossentropy and extended TOPSIS with interval-valued intuitionistic fuzzy sets," Knowledge-Based Systems, vol. 30, pp. 115-120, 2012.

[18] X.-W. Qi, C.-Y. Liang, Y.-Q. Huang, and Y. Ding, "Multiattribute group decision making method based on hybrid evaluation matrix," System Engineering Theory and Practice, vol. 33, no. 2, pp. 473-481, 2013.

[19] B. Farhadinia, "A theoretical development on the entropy of interval-valued fuzzy sets based on the intuitionistic distance and its relationship with similarity measure," Knowledge-Based Systems, vol. 39, pp. 79-84, 2013.

[20] Q.-S. Zhang and S.-Y. Jiang, "A note on information entropy measures for vague sets and its applications," Information Sciences, vol. 178, no. 21, pp. 4184-4191, 2008.

[21] S. M. Chen, "Measures of similarity between vague sets," Fuzzy Sets and Systems, vol. 74, no. 2, pp. 217-223, 1995.

[22] S. Montes, I. Couso, P. Gil, and C. Bertoluzza, "Divergence measure between fuzzy sets," International Journal of Approximate Reasoning, vol. 30, no. 2, pp. 91-105, 2002.

[23] D.-F. Li, “TOPSIS-based nonlinear-programming methodology for multiattribute decision making with interval-valued intuitionistic fuzzy sets," IEEE Transactions on Fuzzy Systems, vol. 18, no. 2, pp. 299-311, 2010.

[24] S. Xiao, "Induced interval-valued intuitionistic fuzzy Hamacher ordered weighted geometric operator and their application to multiple attribute decision making," Journal of Intelligent \& Fuzzy Systems, vol. 27, no. 1, pp. 527-534, 2014.

[25] J. Ye, "Multicriteria fuzzy decision-making method using entropy weights-based correlation coefficients of intervalvalued intuitionistic fuzzy sets," Applied Mathematical Modelling, vol. 34, no. 12, pp. 3864-3870, 2010.

[26] Y. J. Zhang, P. J. Ma, X. H. Su, and C. P. Zhang, "Multi-attribute decision making with uncertain attribute weight information in the framework of interval-valued intuitionistic fuzzy set," Acta Automatica Sinica, vol. 38, no. 2, pp. 220-228, 2012.

[27] Y. Zhang, P. Li, Y. Wang, P. Ma, and X. Su, "Multiattribute decision making based on entropy under interval-valued intuitionistic fuzzy environment," Mathematical Problems in Engineering, vol. 2013, Article ID 526871, 8 pages, 2013.

[28] J.-Q. Wang, K.-J. Li, and H.-Y. Zhang, "Interval-valued intuitionistic fuzzy multi-criteria decision-making approach based on prospect score function," Knowledge-Based Systems, vol. 27, pp. 119-125, 2012.

[29] J. Ye, "A linear programming method based on an improved score function for interval-valued intuitionistic fuzzy multicriteria decision making," The Engineering Economist, vol. 58, no. 3, pp. 179-188, 2013.
[30] X. S. Cai and L. G. Han, "Some induced Einstein aggregation operators based on the data mining with interval-valued intuitionistic fuzzy information and their application to multiple attribute decision making," Journal of Intelligent \& Fuzzy Systems, vol. 27, no. 1, pp. 331-338, 2014.

[31] Y. R. Yang and S. Yuan, "Induced interval-valued intuitionistic fuzzy Einstein ordered weighted geometric operator and their application to multiple attribute decision making," Journal of Intelligent \& Fuzzy Systems, vol. 26, no. 6, pp. 2945-2954, 2014.

[32] J. Ye, "Multicriteria fuzzy decision-making method based on a novel accuracy function under interval-valued intuitionistic fuzzy environment," Expert Systems with Applications, vol. 36, no. 3, pp. 6899-6902, 2009.

[33] G. Wei and G. Lan, "Grey relational analysis method for interval-valued intuitionistic fuzzy multiple attribute decision making," in Proceedings of the 5th International Conference on Fuzzy Systems and Knowledge Discovery (FSKD '08), pp. 291295, Shandong, China, October 2008.

[34] D. G. Park, Y. C. Kwun, J. H. Park, and I. Y. Park, "Correlation coefficient of interval-valued intuitionistic fuzzy sets and its application to multiple attribute group decision making problems," Mathematical and Computer Modelling, vol. 50, no. 9-10, pp. 1279-1293, 2009.

[35] Z.-Y. Bai, "An interval-valued intuitionistic fuzzy TOPSIS method based on an improved score function," The Scientific World Journal, vol. 2013, Article ID 879089, 6 pages, 2013. 


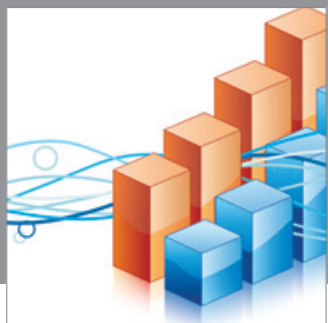

Advances in

Operations Research

mansans

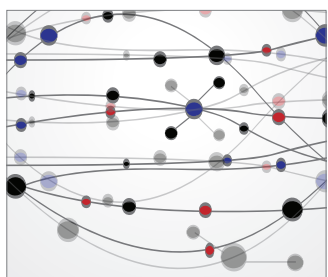

The Scientific World Journal
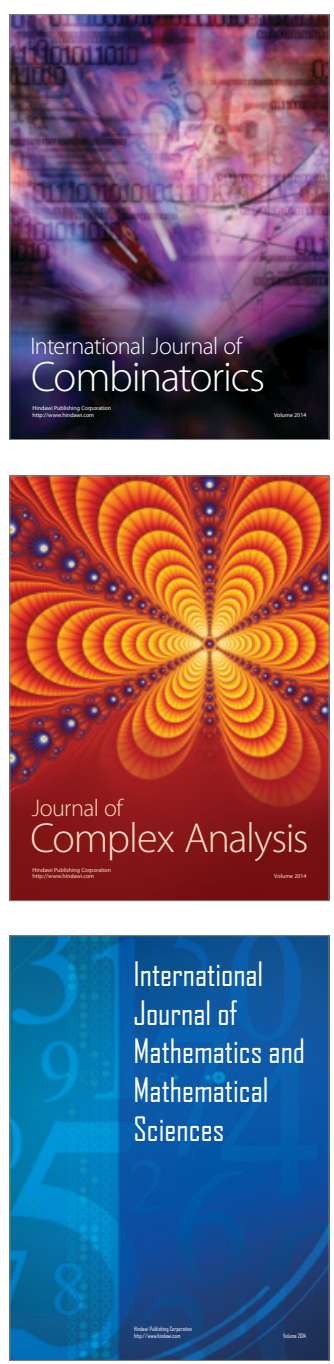
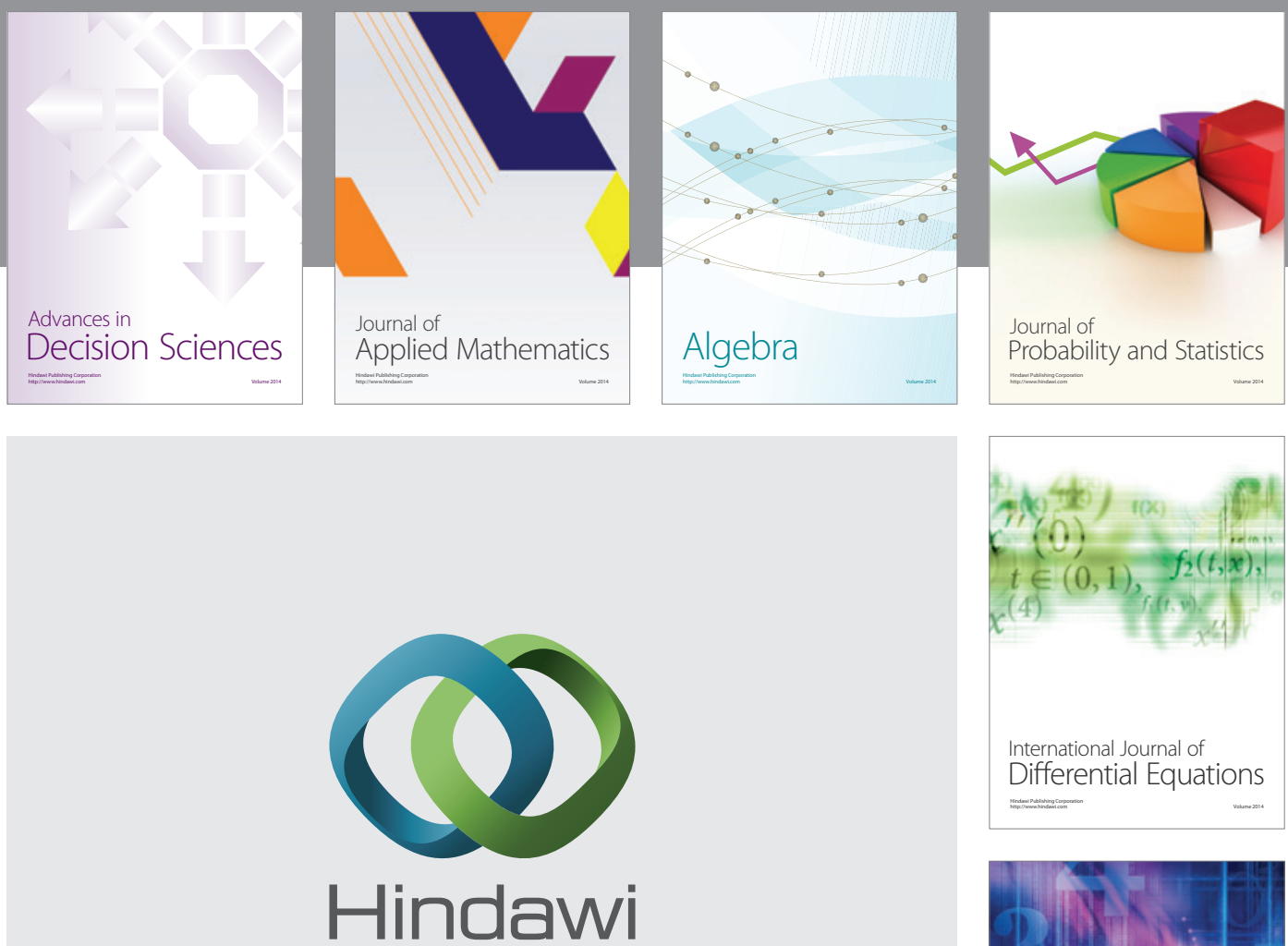

Submit your manuscripts at http://www.hindawi.com
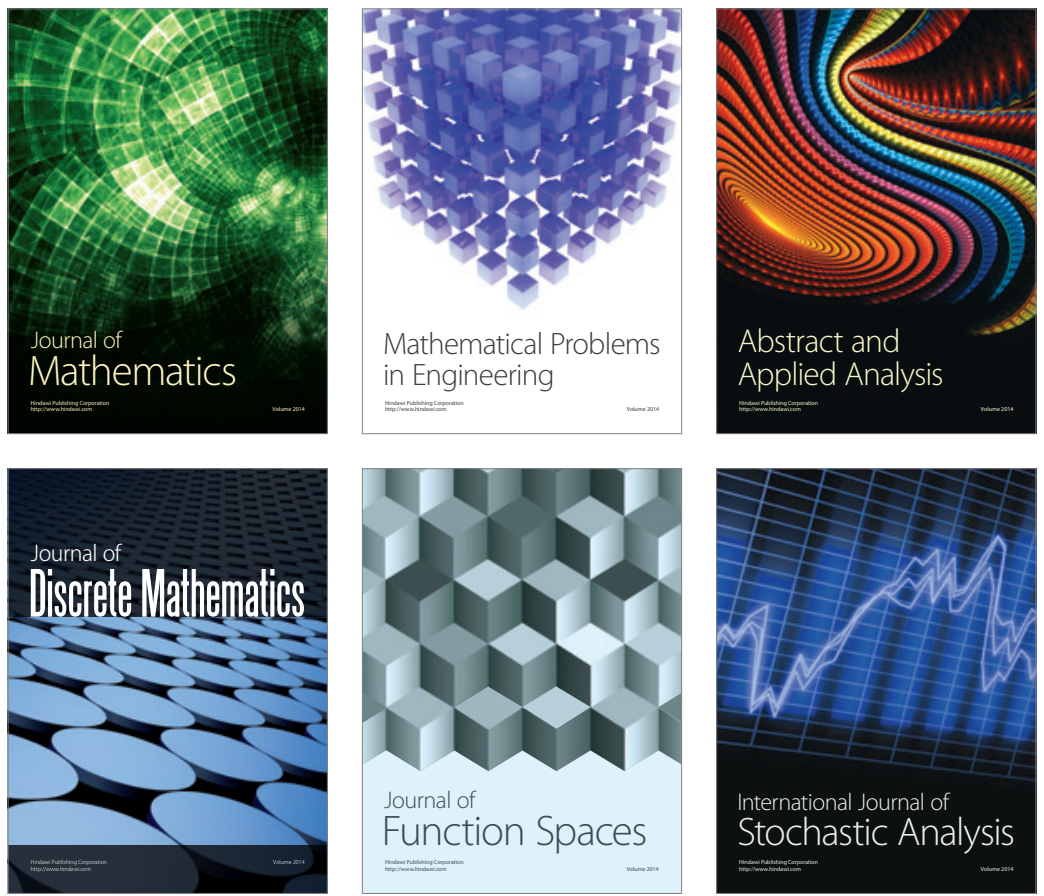

Journal of

Function Spaces

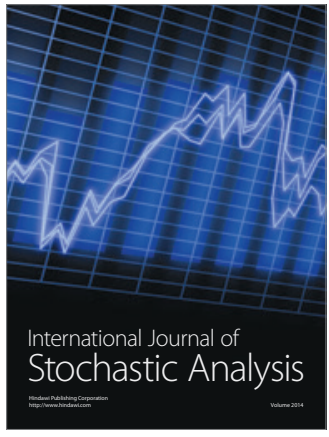

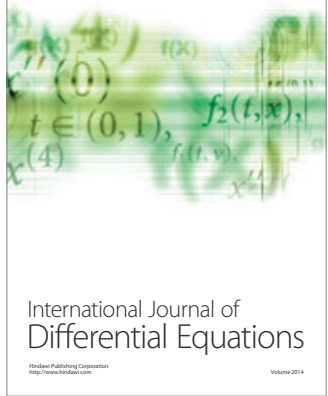
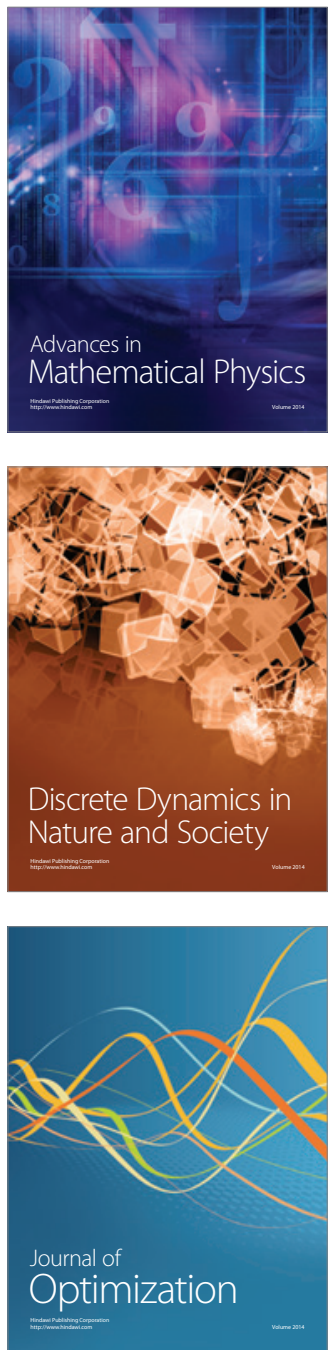